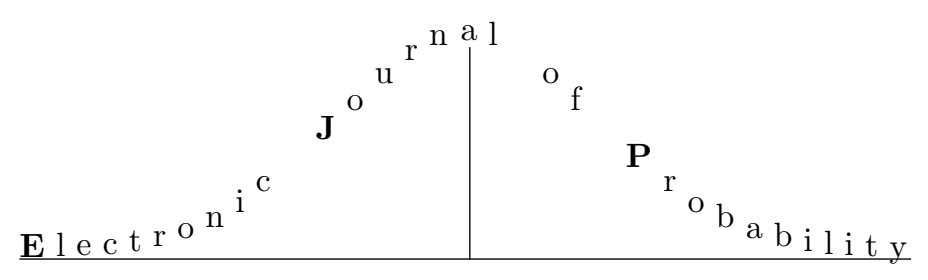

Vol. 13 (2008), Paper no. 7, pages 157-172.

Journal URL

http://www.math.washington.edu/ ejpecp/

\title{
Curve Crossing for the Reflected Lévy Process at zero and infinity
}

\author{
Mladen Savov *
}

\begin{abstract}
Let $R_{t}=\sup _{0 \leq s \leq t} X_{s}-X_{t}$ be a Lévy process reflected in its maximum. We give necessary and sufficient conditions for finiteness of passage times above power law boundaries at infinity. Information as to when the expected passage time for $R_{t}$ is finite, is given. We also discuss the almost sure finiteness of $\limsup _{t \rightarrow 0} R_{t} / t^{\kappa}$, for each $\kappa \geq 0$.
\end{abstract}

Key words: Reflected process, passage times, power law boundaries.

AMS 2000 Subject Classification: Primary 60G51, 60F15, 60K05, 60G40, 60G44.

Submitted to EJP on July 10, 2007, final version accepted January 18, 2008.

${ }^{*}$ School of Mathematics, University of Manchester, Alan Turing Building, Manchester M13 9PL, UK, email: Mladen.S.Savov@postgrad.manchester.ac.uk 


\section{Introduction}

Let $X=\left(X_{t}, t \geq 0\right)$ be a Lévy process starting at zero with characteristic triplet $(\gamma, \sigma, \Pi)$, where $\gamma \in \mathbb{R}, \sigma \geq 0$ and the Lévy measure $\Pi$ has the property $\int_{-\infty}^{\infty} 1 \wedge x^{2} \Pi(d x)<\infty$. We

use $\bar{\Pi}(x)=\int_{y \geq|x|} \Pi(d y)$ to denote the two sided tail of the Lévy measure and $\bar{\Pi}^{(+)}$and $\bar{\Pi}^{(-)}$to denote the corresponding positive and negative tails. Let $\psi(\theta)$ denote the characteristic exponent of $X$, so that

$$
\Psi(\theta)=i \gamma \theta-\frac{\sigma^{2} \theta^{2}}{2}+\int_{-\infty}^{\infty}\left(e^{i \theta x}-1-i \theta x 1_{\{|x| \leq 1\}}\right) \Pi(d x), \text { for all } \theta \in \mathbb{R} .
$$

When $E e^{\lambda X_{1}}$ exists, for all $\lambda$ in an open interval containing 0, we can extend $\Psi$ analytically in some neighbourhood of the real line in the complex plane and refer to the Laplace exponent $\psi$, which relates to $\Psi$ via the identity

$$
\psi(\theta)=\ln E e^{\theta X_{1}}=-\Psi(-i \theta)
$$

For any Lévy process we can define the reflected process $R=\left(R_{t}\right)_{t \geq 0}$ as follows:

$$
R_{t}=\bar{X}_{t}-X_{t}, \text { for any } t \geq 0,
$$

where $\bar{X}_{t}=\sup _{0 \leq s \leq t} X_{s}$. We note that whenever we have the notation $\bar{Y}_{t}$, we mean $\bar{Y}_{t}=$ $\sup _{s \in I \cap[0, t]} Y_{s}$, where $I$ is either $\mathbb{R}_{+}$or $\mathbb{Z}_{+}$.

The reflected process plays an important role in the theory of random walks and Lévy processes, and has many applications in finance, genetics and optimal stopping. Thus, for example, the optimal time to exercise "Russian option" is the first time the reflected process crosses a fixed level (Shepp and Shiryaev [13], [14] and Asmussen, Avram and Pistorius). For more discussions and basic properties of the reflected process we refer to [5].

The first aim of this paper is to obtain necessary and sufficient conditions (NASC) for the almost sure (a.s.) finiteness of passage times of $R_{t}$ out of power law regions of the form $\left[0, r t^{\kappa}\right]$ where $r>0$ and $\kappa \geq 0$, and for the finiteness of the expected value of passage times of $R_{t}$ from linear $(\kappa=1)$ or parabolic $(\kappa=1 / 2)$ regions. We also provide a NASC for the a.s. finiteness of $\lim \sup _{t \rightarrow 0} R_{t} / t^{\kappa}$, for any $\kappa \geq 0$.

Section 2 essentially extends results for random walks in [5]. We obtain NASC for when $\limsup _{t \rightarrow \infty} R_{t} / t^{\kappa}$ is a.s. finite or not, for any $\kappa \in \mathbb{R}_{+}$. To achieve this we rely on very useful stochastic bounds discovered recently by Doney in [7]. The Section is completed by discussing the finiteness of expected values of passage times of $R_{t}$.

In Section 3 new results for the passage time of $R_{t}$ at 0 are obtained. The NASC are very similar to the ones at $\infty$. It turns out that the integrability of the Lévy measure ( see Theorem $3.1(i)$ and Theorem $3.2(i i)$ ) plays the same role as the finiteness of particular moments of the Lévy process ( see Theorem 2.1 (b)).

The proofs are given in Section 4 and Section 5, while some technical results are collected in the Appendix. 


\section{Passage times above power law boundaries at infinity}

In [5], results about the first exit time of a reflected random walk from power law regions are obtained. These include NASC for a.s. finiteness of both the the first exit time and its expectation. In this Section we extend these results to reflected Lévy processes. The main technique in proving Theorem 2.1 is the stochastic bound discovered recently by Doney, see [7]. It is possible to derive this result using a standard embedded random walk $\widehat{X}:=\left(X_{n} ; n \geq 0\right)$, where $X_{n}$ is the Lévy process computed at time $n$. We prove Theorem 2.2 by using functions of $R_{t}$ which define martingales on $\mathbb{R}_{+}$.

We define, for any $\kappa \geq 0$ and $r>0$ :

$$
\tau_{\kappa}(r)=\inf \left\{t \geq 0: R_{t}>r(t+1)^{\kappa}\right\},
$$

where $t+1$ is used for $t$ to avoid the case when $\tau_{\kappa}(r)=0$ a.s. Let $X^{+}=X_{+}=\max \{X, 0\}$ and $X^{-}=X_{-}=\max \{-X, 0\}$. We may now state our main result.

Theorem 2.1. (a) Suppose $\kappa=0$. Then $\tau_{0}(r)=\tau(r)<\infty$ a.s. for all $r \geq 0$ iff $X$ is not a positive subordinator. Moreover for each $r>0$, there is $\lambda(r)>0$, such that $E e^{\lambda \tau(r)}<\infty$, for all $\lambda \leq \lambda(r)$.

(b) Suppose $\kappa>0$. We have $\tau_{\kappa}(r)<\infty$ a.s., for all $r>0$, iff

(i) for $\kappa>1: E\left(X_{1}^{-}\right)^{1 / \kappa}=\infty$;

(ii) for $0<\kappa \leq 1$ : $E\left(X_{1}^{-}\right)^{1 / \kappa}=\infty$ or $\liminf _{t \rightarrow \infty} \frac{X_{t}}{t^{\kappa}}=-\infty$ a.s.

Remarks. (i) Note that $\tau_{\kappa}(r)<\infty$ a.s., for all $r>0$, is equivalent to

$$
\limsup _{t \rightarrow \infty} \frac{R_{t}}{t^{\kappa}}=\infty \text { a.s. }
$$

This may not seem obvious, but can be proved in the same way as in Lemma 3.1. in [5]. For an alternative proof see [12].

(ii) Also note, that for the embedded random walk $\widehat{X}=\left(X_{n}, n \geq 0\right)$, the following inequality holds

$$
R_{n}^{\widehat{X}} \leq R_{n}
$$

where $R^{\widehat{X}}$ is the reflected process for $\widehat{X}$. This implies that

$$
\tau_{\kappa}^{\widehat{X}}(r) \geq \tau_{\kappa}(r),
$$

for any $\kappa \geq 0$ and $r>0$.

(iii) We exclude the case of positive subordinator since then $R_{t} \equiv 0$. In this case obviously $\tau_{\kappa}(r)=\infty$ a.s.

(iv) For analytic conditions equivalent to $\lim _{\inf } \operatorname{li}_{t \rightarrow \infty} \frac{X_{t}}{t^{\kappa}}=-\infty$ a.s. we refer to [6].

The second result considers the expected value of the passage time of $R_{t}$ above linear and square root boundaries and extends the corresponding result in [5].

Theorem 2.2. (a) Suppose $E X_{1}^{2}=\alpha^{2}<\infty$ and $E X_{1}=0$. Then 
(i) $E \tau_{1 / 2}(\alpha r)<\infty$, for all $r<1$;

(ii) $E \tau_{1 / 2}(\alpha r)=\infty$, for all $r \geq 1$.

(b) Suppose $E X_{1}<0, E\left|X_{1}\right|<\infty$ and $E\left(X_{1}^{+}\right)^{2}<\infty$. Then

(i) $E \tau_{1}(r)<\infty$, for all $r<-E X_{1}$;

(ii) $E \tau_{1}(r)=\infty$, for all $r \geq-E X_{1}$.

Remarks. (i) The general approach to estimate the expectation of the first exit time is via functions of $R_{t}$ that define martingales on $\mathbb{R}_{+}$, see Theorem 2.2. in [5]. This constrains us to linear and square root boundaries and it is not clear how this approach could be extended for a general boundary when $\frac{1}{2}<\kappa<1$.

(ii) Despite some efforts, we have been unable to remove the restriction $E\left(X_{1}^{+}\right)^{2}<\infty$ in (b). Generally, it seems to be difficult to obtain results for the finiteness of the expected values of the passage times when $E X^{2}=\infty$, not only for the reflected process, but for random walks as well. For short discussion we refer to [5].

\section{Passage times above power law boundaries at zero}

In this Section we discuss passage times of the reflected process above power law boundaries at zero. To avoid notational complications we will study

$$
\limsup _{t \rightarrow 0} \frac{R_{t}}{t^{\kappa}}=\infty \text { a.s. }
$$

rather than the equivalent condition

$$
\widetilde{\tau}_{\kappa}(r)=\inf \left\{t>0: R_{t}>r t^{\kappa}\right\}=0 \text { a.s., for all } r>0 \text {. }
$$

The first theorem deals with Lévy processes with bounded variation.

Theorem 3.1. Let $X$ be a Lévy process with bounded variation and drift $d$, defined by $\lim _{t \rightarrow 0} X_{t} / t=d$ a.s. Then the following statements hold

(i) For $\kappa>1$, (3.1) holds iff either

$$
\int_{0}^{1} \bar{\Pi}^{(-)}\left(x^{\kappa}\right) d x=\infty \text { or } d<0 .
$$

(ii) For $\kappa \leq 1$, we have

(a) If $\kappa<1$, or, if $\kappa=1$ and $d \geq 0$, then

$$
\lim _{t \rightarrow 0} \frac{R_{t}}{t^{\kappa}}=0 \text { a.s. }
$$


(b) If $\kappa=1$ and $d<0$, then

$$
\lim _{t \rightarrow 0} \frac{R_{t}}{t}=-d \text { a.s. }
$$

Next we deal with the unbounded variation case. We have the following result:

Theorem 3.2. Let $X$ be a Lévy process with unbounded variation.

(i) If $\kappa \geq 1$, then (3.1) holds.

(ii) If $1 / 2 \leq \kappa<1$, then (3.1) holds iff

$$
\begin{aligned}
& \text { (A) } \int_{0}^{1} \bar{\Pi}^{(-)}\left(x^{\kappa}\right) d x=\infty \text { or } \\
& \text { (B) } \liminf _{t \rightarrow 0} \frac{X_{t}}{t^{\kappa}}=-\infty \text { a.s. }
\end{aligned}
$$

(iii) If $\kappa<1 / 2$, then

$$
\lim _{t \rightarrow 0} \frac{R_{t}}{t^{\kappa}}=0 \text { a.s. }
$$

Remarks. (i) Now it is worth mentioning the similarity between Theorem 3.1 (ii), Theorem 3.2 (ii) and Theorem 2.1 (b). The integrability of the negative Lévy tail is directly comparable to the finiteness of $E\left(X_{1}^{-}\right)^{1 / \kappa}$.

(ii) It needs to be mentioned, that (A) and (B) in (ii) are not equivalent. For example, if $\kappa=1 / 2$, (A) fails while (B) can happen, see Theorem 2.2. in [3]. Moreover, for $\frac{1}{2}<\kappa<1$, $\int_{0}^{1} \bar{\Pi}\left(x^{\kappa}\right) d x<\infty$ implies that $\liminf _{t \rightarrow 0} \frac{X_{t}}{t^{\kappa}}=0$ a.s., see Theorem 2.1. in [3].

(iii) Analytic conditions for $\liminf _{t \rightarrow 0} \frac{X_{t}}{t^{\kappa}}=-\infty$ a.s. can be found in [3].

\section{Proofs for section 2}

Proof of Theorem 2.1. We start with the proof of (a). If $X$ is a negative subordinator, then $R_{t}=-X_{t}$ and the statement that $\tau(r)<\infty$ a.s. is clear from the fact that $X_{t}$ drifts to $-\infty$. Without loss of generality, we assume that $\bar{\Pi}^{(-)}(1)>0$. Obviously

$$
\tau(r)<\wp(r)=\inf \{t: X \text { has jumped }[r]+1 \text { times with jumps less than }-1\} .
$$

Since $\wp(r)$ is a sum of independent exponentially distributed random variables it has gamma distribution and hence $E e^{\lambda \tau(r)} \leq E e^{\lambda \wp(r)}<\infty$, for $\lambda$ small enough.

To show $E e^{\lambda \tau(r)}<\infty$, for some $\lambda>0$, for a general Lévy process, we invoke Theorem 2.1 (a) in [5] for the embedded random walk defined in remark (ii) of Theorem 2.1 and use inequality (2.4).

We shall prove the forward part of both $(i)$ and $(i i)$ in $(b)$ together. Assume (2.2) holds.

Denote by $\left\{\zeta_{i}\right\}_{i \geq 0}$ the stopping times defined recursively by

$$
\zeta_{i+1}=\inf \left\{t>\zeta_{i}:\left|\Delta X_{t}\right|>1\right\} \text { and } \zeta_{0}=0
$$


We use Theorem 1.1 in [7] to construct a stochastic bound $M_{n}$ for $X_{t}$ with the following property:

$$
X_{t} \leq M_{n}=\sup _{\zeta_{n} \leq t<\zeta_{n+1}} X_{t}=S_{n}^{+}+m_{0}, \text { for } \zeta_{n} \leq t<\zeta_{n+1}
$$

where $S_{n}^{+}$is a random walk with steps

$$
Y_{i}=X_{\zeta_{i}}-\sup _{\zeta_{i-1} \leq t<\zeta_{i}} X_{t}+\sup _{\zeta_{i} \leq t<\zeta_{i+1}}\left(X_{t}-X_{\zeta_{i}}\right)
$$

and $m_{0}=\sup _{t \leq \zeta_{1}} X_{t}$. In fact $Y_{i}$ can be represented in the following useful way

$$
Y_{i}=J_{i}+\widetilde{X}_{\zeta_{i}}-\sup _{\zeta_{i-1} \leq t<\zeta_{i}} \widetilde{X}_{t}+\sup _{\zeta_{i} \leq t<\zeta_{i+1}} \widetilde{X}_{t}-\widetilde{X}_{\zeta_{i}} \stackrel{d}{=} J_{1}+\widetilde{X}_{\zeta_{1}}
$$

where $J_{i}=\Delta X_{\zeta_{i}}=X_{\zeta_{i}}-X_{\zeta_{i}-}$, and $\tilde{X}$ is obtained from $X$ by removing all jumps bigger in absolute value than 1 . Then the Lévy measure of $\tilde{X}$ has compact support and hence from Theorem 25.17 in [11], for example, we have that $E e^{\lambda \widetilde{X}}<\infty$, for all $\lambda>0$.

With $N(t):=\max \left\{i: \zeta_{i} \leq t\right\}$ and $\bar{M}_{N(t)}=\max _{n \leq N(t)} M_{n}$, we have the following inequality

$$
R_{t}=\bar{X}_{t}-X_{t} \leq \bar{M}_{N(t)}-M_{N(t)}+M_{N(t)}-X_{t}
$$

Recall that $m_{0} \geq 0$ a.s. and therefore the reflected random walk of $S^{+}$has the form $R_{N(t)}^{+}=$ $\bar{M}_{N(t)}-M_{N(t)}=\bar{S}_{N(t)}^{+}-S_{N(t)}^{+}$. Moreover by (4.1) and (4.2) we get $M_{N(t)}-X_{t}=m_{0}+\widetilde{S}_{N(t)}^{+}-\widetilde{X}_{t}$, where $\widetilde{S}_{n}^{+}=S_{n}^{+}-\sum_{k \leq n} J_{k}$. These observations enable the following useful upper bound for $R_{t}$ :

$$
R_{t} \leq R_{N(t)}^{+}+m_{0}+\widetilde{S}_{N(t)}^{+}-\widetilde{X}_{t}
$$

We now show that

$$
\limsup _{t \rightarrow \infty} \frac{m_{0}+\widetilde{S}_{N(t)}^{+}-\widetilde{X}_{t}}{t^{\kappa}}=0 \text { a.s. }
$$

Indeed note that since $m_{0}+\widetilde{S}_{N(t)}^{+}=\sup _{\zeta_{N(t)} \leq s<\zeta_{N(t)+1}} \widetilde{X}_{s}$, we immediately have that

$$
\begin{aligned}
& \limsup _{t \rightarrow \infty} \frac{m_{0}+\widetilde{S}_{N(t)}^{+}-\widetilde{X}_{t}}{t^{\kappa}} \leq \limsup _{t \rightarrow \infty} \frac{2 \sup _{\zeta_{N(t)} \leq s<\zeta_{N(t)+1}}\left|\widetilde{X}_{s}-\widetilde{X}_{\zeta_{N(t)}}\right|}{t^{\kappa}}= \\
& \lim _{t \rightarrow \infty} \frac{N(t)^{\kappa}}{t^{\kappa}} \limsup _{n \rightarrow \infty} \frac{2 \sup _{\zeta_{n} \leq s<\zeta_{n+1}}\left|\widetilde{X}_{s}-\widetilde{X}_{\zeta_{n}}\right|}{n^{\kappa}} \leq \\
& C \limsup _{n \rightarrow \infty} \frac{2 \sup _{\zeta_{n} \leq s<\zeta_{n+1}}\left|\widetilde{X}_{s}-\widetilde{X}_{\zeta_{n}}\right|}{n^{\kappa}}
\end{aligned}
$$

where $\lim _{t \rightarrow \infty} \frac{N(t)^{\kappa}}{t^{\kappa}}=C>0$ a.s. follows by the strong law of large numbers. Now set $V_{n}=\sup _{\zeta_{n} \leq s<\zeta_{n+1}}\left|\widetilde{X}_{s}-\widetilde{X}_{\zeta_{n}}\right|$ and observe that $\left\{V_{i}\right\}_{i \geq 0}$ are mutually independent and $V_{n} \stackrel{d}{=} V_{0}$. Therefore to get (4.4) we simply need

$$
\limsup _{n \rightarrow \infty} \frac{V_{n}}{n^{\kappa}}=0 \text { a.s. }
$$


To achieve this recall that $\widetilde{X}_{t}$, as well as $\zeta_{1}$, have finite moments of any order (recall that Theorem 25.17 in [11] implies $E e^{\lambda X_{t}}<\infty$, for any $\lambda \in \mathbb{R}$ ). This easily implies that $V_{0}$ has moments of any order and hence, for any $\varepsilon>0$,

$$
\sum_{n \geq 0} P\left(V_{n}>\varepsilon n^{\kappa}\right)=\sum_{n \geq 0} P\left(V_{0}>\varepsilon n^{\kappa}\right)<\infty
$$

A simple application of the Borel-Cantelli lemma yields (4.5) and hence (4.4). Lastly we see that (2.2) and (4.3) along with the strong law of large numbers give

$$
\limsup _{t \rightarrow \infty} \frac{R_{N(t)}^{+}}{t^{\kappa}}=C \limsup _{n \rightarrow \infty} \frac{R_{n}^{+}}{n^{\kappa}}=\infty \text { a.s. }
$$

All that remains is to apply Theorem 2.1. in [5] to the random walk $S_{n}^{+}$and deduce that either $E\left(Y_{-}\right)^{\frac{1}{\kappa}}=\infty$, for any $\kappa \geq 0$, or

$$
\liminf _{n \rightarrow \infty} \frac{S_{n}^{+}}{n^{\kappa}}=-\infty, \text { when } \kappa \leq 1
$$

Then the definition of $Y$ implies that $E X_{-}^{\frac{1}{\kappa}}=\infty$ in case $E Y_{-}^{\frac{1}{\kappa}}=\infty$, and similarly $\liminf _{n \rightarrow \infty} \frac{S_{n}^{+}}{n^{\kappa}}=-\infty$ a.s. implies $\liminf _{t \rightarrow \infty} \frac{X_{t}}{t^{\kappa}}=-\infty$ a.s.

The backward part of $(b)$ is much simpler since we can directly use $R_{t} \geq-X_{t}$ when $\liminf _{t \rightarrow 0} X_{t} / t^{\kappa}=-\infty$, or apply Theorem 2.1. in [5] to the embedded random walk $\widehat{X}$, when $E\left(X_{1}^{-}\right)^{1 / \kappa}=\infty$. We therefore see that

$$
\limsup _{n \rightarrow \infty} \frac{R_{n}^{\widehat{X}}}{n^{\kappa}}=\infty \text { a.s. }
$$

where $R^{\widehat{X}}$ is the reflected random walk for $\widehat{X}$ and applying (2.3) we conclude the proof.

Proof of Theorem 2.2. Part (i) for both (a) and (b) follow easily from Theorem 2.2 in [5] together with inequality (2.4). We therefore concentrate on (ii), (a). Observe that since $E X_{1}=0, X_{t}$ is a martingale. Also note that the maximum process $\bar{X}_{t}$ has bounded variation and therefore $R_{t}$ is a semimartingale. Moreover $E X_{t}^{2}<\infty$ implies that $E \bar{X}_{t}^{2}<\infty$, see Theorem 25.18 in [11], which in turn gives $E R_{t}^{2}<\infty$.

Denoting by $[.]_{t}$ the quadratic variation of a process and applying Itô's formula, see [9], p.71, we see that

$$
R_{t}^{2}=2 \int_{0}^{t} R_{s-} \mathrm{d} R_{s}+[R]_{t}=[R]_{t}+2 \int_{0}^{t} R_{s-} \mathrm{d} \bar{X}_{s}-2 \int_{0}^{t} R_{s-} \mathrm{d} X_{s} .
$$

Now by virtue of the fact that $\bar{X}$ has bounded variation, it follows that

$$
\begin{gathered}
{[R]_{t}=[\bar{X}]_{t}-2[\bar{X}, X]_{t}+[X]_{t}=[X]_{t}-\sum_{s \leq t}\left(2 \Delta \bar{X}_{s} \Delta X_{s}-\Delta \bar{X}_{s}^{2}\right)} \\
R_{t}^{2}=[X]_{t}-\sum_{s \leq t}\left(2 \Delta \bar{X}_{s} \Delta X_{s}-\Delta \bar{X}_{s}^{2}\right)+2 \int_{0}^{t} R_{s-} \mathrm{d} \bar{X}_{s}-2 \int_{0}^{t} R_{s-} \mathrm{d} X_{s} .
\end{gathered}
$$


For $\mathrm{P}$ a.e. $\omega$ in $\Omega$, we have $\bar{X}_{t}(\omega)=\sum_{s \leq t} \Delta \bar{X}_{t}(\omega)+G(t, w)$, where the function $G(., \omega)$ is nonnegative, nondecreasing and continuous. This follows from the fact that for any given $\omega, t \mapsto \bar{X}_{t}(\omega)$ is a right continuous, nondecreasing and nonnegative function. Consequently we see that then

$$
\int_{0}^{t} R_{s-} \mathrm{d} \bar{X}_{s}(\omega)=\sum_{s \leq t} \Delta \bar{X}_{s}(\omega) R_{s-}(\omega)+\int_{0}^{t} R_{s-}(\omega) \mathrm{d} G(s, w) .
$$

In Proposition 6.1, see Appendix, we show that

$$
\int_{0}^{t} R_{s-}(\omega) \mathrm{d} G(s, \omega)=0, \text { a.s. for all } t \geq 0 .
$$

Inserting this into (4.8) above and substituting (4.8) into (4.7), we obtain

$$
R_{t}^{2}=[X]_{t}-\sum_{s \leq t}\left(2 \Delta \bar{X}_{s} \Delta X_{s}-\Delta \bar{X}_{s}^{2}-2 \Delta \bar{X}_{s} R_{s-}\right)-2 \int_{0}^{t} R_{s-} \mathrm{d} X_{s}
$$

Since we have

$$
\begin{gathered}
\Delta R_{s}=R_{s}-R_{s-}=-\left(\Delta X_{s} 1_{\left\{\Delta X_{s} \leq R_{s-}\right\}}+R_{s-} 1_{\left\{\Delta X_{s}>R_{s-}\right\}}\right) \\
\Delta \bar{X}_{s}=\Delta R_{s}+\Delta X_{s}=\left(\Delta X_{s}-R_{s-}\right) 1_{\left\{\Delta X_{s}>R_{s-}\right\}},
\end{gathered}
$$

we may insert these identities into (4.9) to deduce that

$$
R_{t}^{2}=[X]_{t}-\sum_{s \leq t}\left(\Delta X_{s}-R_{s-}\right)^{2} 1_{\left\{\Delta X_{s}>R_{s-}\right\}}-2 \int_{0}^{t} R_{s-} \mathrm{d} X_{s} .
$$

We are ready now to conclude the proof of the theorem. First we note that $\int_{0}^{t} R_{s-} \mathrm{d} X_{s}$ and $X_{t}^{2}-[X]_{t}$ are zero mean martingales. Then we apply the optional sampling theorem to the last identity to get

$$
E R_{\tau_{1 / 2}(\alpha r) \wedge m}^{2}-\alpha^{2} E \tau_{1 / 2}(\alpha r) \wedge m+E\left(\sum_{s \leq \tau_{1 / 2}(\alpha r) \wedge m}\left(\Delta X_{s}-R_{s-}\right)^{2} 1_{\left\{\Delta X_{s}>R_{s-}\right\}}\right)=0
$$

for any $m>0$, and hence

$$
E R_{\tau_{1 / 2}(\alpha r) \wedge m}^{2} \leq \alpha^{2} E \tau_{1 / 2}(\alpha r) \wedge m
$$

If we assume that $E \tau_{1 / 2}(\alpha r)<\infty$, we see from Fatou's lemma and the definition of $\tau$. $(r)$ that

$$
\liminf _{m \rightarrow \infty}\left(E\left(R_{\tau_{1 / 2}(\alpha r) \wedge m}\right)^{2}\right) \geq E\left(R_{\tau_{1 / 2}(\alpha r)}^{2}\right)>r^{2} \alpha^{2}\left(E \tau_{1 / 2}(\alpha r)+1\right) .
$$

Applying the monotone convergence theorem we deduce

$$
\lim _{m \rightarrow \infty} E\left(\tau_{1 / 2}(\alpha r) \wedge m\right)=E\left(\tau_{1 / 2}(\alpha r)\right)
$$

and this together with (4.10) gives $\left(1-r^{2}\right) \alpha^{2} E \tau_{1 / 2}(\alpha r)>r^{2} \alpha^{2}>0$. Since this is an obvious contradiction when $r \geq 1$, we see that we must have $E \tau_{1 / 2}(\alpha r)=\infty$, for all $r \geq 1$. 
Turning now to the proof of part $(b),(i i)$, we can assume, without loss of generality, that $E X_{1}=-1$. From [12] and $E\left(X_{1}^{+}\right)^{2}<\infty$ we see that $l=E \bar{X}_{\infty}<\infty$. Define the exit times,

$$
T_{q}=\inf \left\{t>0: R_{t}>t+q\right\},
$$

for all $q \geq 1$. Assume that $E T_{q}<\infty$, for each $q \geq 1$. An easy application of the optional sampling theorem to $X_{T_{q} \wedge m}$, followed by the monotone convergence theorem and Fatou's lemma, yields

$$
E T_{q}+q \leq E R_{T_{q}} \leq \lim _{m \rightarrow \infty} E R_{T_{q} \wedge m}=E \bar{X}_{T_{q}}+E T_{q} \leq l+E T_{q}
$$

Therefore we must have $E T_{q}=\infty$ when $q>l$. Next observe that, for each $q \geq 1$,

$$
\begin{aligned}
& E T_{q} 1_{\left\{T_{q}>1\right\}} \geq \int_{0}^{1-\varepsilon} E\left(T_{q} \mid R_{1}=y, T_{q}>1\right) P\left(R_{1} \in d y, T_{q}>1\right) \geq \\
& \int_{0}^{1-\varepsilon} E\left(T_{1+q-y}\right) P\left(R_{1} \in d y, T_{q}>1\right) \geq E T_{q+\varepsilon} P\left(R_{1} \in(0,1-\varepsilon), T_{1}>1\right) .
\end{aligned}
$$

The first inequality comes from narrowing the possible values of $R_{1}$, while the second, which reads $E\left(T_{q} \mid R_{1}=x, T_{q}>1\right) \geq E T_{q-x}$, for $x \in(0,1)$, is verified using the fact that $R_{t}$ is a Markov process. If we assume that $X$ is not a negative drift, then $\exists \delta>0: P\left(R_{1} \in(0,1-\varepsilon), T_{1}>1\right)>\delta$. This implies that

$$
E T_{q} 1_{\left\{T_{q}>1\right\}} \geq \delta E T_{q+\varepsilon} \geq \delta E T_{q+\varepsilon} 1_{\left\{T_{q+\varepsilon}>1\right\}},
$$

and repeating this step finitely many times, we get

$$
E T_{q} 1_{\left\{T_{q}>1\right\}}>C E T_{2 l} 1_{\left\{T_{2 l}>1\right\}}=\infty,
$$

where $\mathrm{C}$ is some constant. Therefore we must have $E T_{1}=\infty$.

\section{$5 \quad$ Proofs for Section 3}

First of all, we observe that for a study of the behaviour at zero we can always assume that the Lévy measure is carried by $[-1,1]$. With this in mind we proceed with the proof of Theorem 3.1. Recall that, since $X$ has bounded variation, we can write

$$
X_{t}=d t+Y_{t}+Z_{t}
$$

where $Y$ is a driftless positive subordinator and $Z$ is a driftless negative subordinator. To show (i), let us first suppose that

$$
\int_{0}^{1} \bar{\Pi}^{(-)}\left(x^{\kappa}\right) d x<\infty
$$

Then applying Theorem 9, Chapter 3 in [2] to $-Z_{t}$ in (5.1), we easily get

$$
\lim _{t \rightarrow 0} \frac{Z_{t}}{t^{\kappa}}=0 \text { a.s. }
$$


Therefore, if $d \geq 0$ we have the bound

$$
R_{t} \leq \sup _{s \leq t}\left(Y_{s}+d s\right)-Y_{t}-d t-Z_{t}=-Z_{t}
$$

and (3.1) fails. However, if $d<0$, we know that $\lim _{t \rightarrow 0} \frac{X_{t}}{t}=d$ a.s., and hence $\lim _{t \rightarrow 0} \frac{X_{t}}{t^{\kappa}}=$ $-\infty$ a.s. Then by the simple inequality $R_{t} \geq-X_{t}$ we deduce (3.1).

Assume now that (5.2) fails. Then a standard argument, see Theorem 9 on page 85 of [2], gives, for any $c>1,-\Delta X_{t}>c t^{\kappa}$ i.o., which along with $R_{t} \geq-\Delta X_{t} 1_{\left\{\Delta X_{t}<0\right\}}$ shows that (3.1) holds. For $(i i),(a)$, all we need to observe is that from (5.1) we have

$$
R_{t} \leq \sup _{s \leq t}\left(Y_{s}+d s\right)-Y_{t}-d t-Z_{t} \leq 0 \vee-d t-Z_{t}
$$

and recall $\lim _{t \rightarrow 0} \frac{Z_{t}}{t}=0$ a.s.

For $(i i)$, (b), we note that

$$
\lim _{t \rightarrow 0} \frac{\sup _{s \leq t} X_{s}}{t} \leq \lim _{t \rightarrow 0} \frac{Y_{t}-Z_{t}}{t}=0 \text { a.s. }
$$

and therefore

$$
\lim _{t \rightarrow 0} \frac{R_{t}}{t}=\lim _{t \rightarrow 0} \frac{\sup _{s \leq t} X_{s}-X_{t}}{t}=\lim _{t \rightarrow 0} \frac{-X_{t}}{t}=-d \text { a.s. }
$$

This completes the proof of Theorem 3.1 .

Proof of Theorem 3.2. For $\kappa \geq 1$, we invoke a standard result of Rogozin, see [10], to show that $\liminf _{t \rightarrow 0} \frac{X_{t}}{t}=-\infty$ a.s.

For (iii) we use a result of Khintchine, see [8], stating that:

$$
\limsup _{t \rightarrow 0} \frac{X_{t}}{\sqrt{2 t \ln |\ln t|}}=\limsup _{t \rightarrow 0} \frac{-X_{t}}{\sqrt{2 t \ln |\ln t|}}=\sigma \text { a.s. . }
$$

Hence (3.1) fails since $\sqrt{2 t \ln |\ln t|}=o\left(t^{\kappa}\right)$, for all $\kappa<1 / 2$, as $t$ goes to 0 . The same way we see that, for $\kappa=1 / 2$ and $\sigma>0$, (3.1) fails and that (3.1) holds, for $1 / 2<\kappa<1$ and $\sigma>0$.

From now on we set $\sigma=0$ and proceed with part (ii). Suppose first that $\kappa=1 / 2$, so that (A) fails. In view of Theorem 2.2. in [3], we have that either

$$
\liminf _{t \rightarrow \infty} \frac{X_{t}}{\sqrt{t}}=-\infty \text { a.s., }
$$

which is exactly condition (B) and mean that (3.1) holds, or

$$
\limsup _{t \rightarrow 0} \frac{\left|X_{t}\right|}{\sqrt{t}}<\infty \text { a.s. }
$$

which means that (3.1) and (B) fail simultaneously. 
For $1 / 2<\kappa<1$, if $\int_{0}^{1} \bar{\Pi}\left(x^{\kappa}\right) d x<\infty$, then in view of Theorem 2.1 in [3], we see that (B) and (3.1) fail, while if $\int_{0}^{1} \bar{\Pi}^{(-)}\left(x^{\kappa}\right) d x=\infty$, then Theorem 3.1 in [3] implies that (3.1) holds since $\liminf _{t \rightarrow 0} \frac{X_{t}}{t^{\kappa}}=-\infty$ a.s. It remains to consider the case

$$
\int_{0}^{1} \bar{\Pi}^{(-)}\left(x^{\kappa}\right) d x<\infty=\int_{0}^{1} \bar{\Pi}^{(+)}\left(x^{\kappa}\right) d x .
$$

Write $X=X^{+}+X^{-}$as a sum of two independent Lévy processes, where $X^{+}$is a zero mean, spectrally positive Lévy process and $X^{-}$is a zero mean, spectrally negative Lévy process. Then since $\int_{0}^{1} \bar{\Pi}^{(-)}\left(x^{\kappa}\right) d x<\infty$, we can apply Theorem 2.1, Proposition 4.1 and Proposition 4.2 in [3] to deduce that

$$
\lim _{t \rightarrow 0} \frac{\sup _{s \leq t}\left|X_{s}^{-}\right|}{t^{\kappa}}=0 \text { a.s. }
$$

and thus

$$
\limsup _{t \rightarrow 0} \frac{R_{t}}{t^{\kappa}}=\limsup _{t \rightarrow 0} \frac{R_{t}^{+}}{t^{\kappa}}, \text { a.s. }
$$

where $R_{t}^{+}=\bar{X}_{t}^{+}-X_{t}^{+}$. Therefore we may additionally assume that $X$ is a zero mean, spectrally positive Lévy process and continue with the proof. Let us define the functions

$$
\begin{gathered}
V(x):=\int_{0}^{x} y^{2} \Pi(d y), \\
W(x):=\int_{0}^{x} \int_{z}^{1} s \Pi(d s) d z=V(x)+x \int_{x}^{1} s \Pi(d s),
\end{gathered}
$$

for all $x \geq 0$, so that $W(x)$ is continuous and nondecreasing. For any $\lambda>0$, we now define the function

$$
J(\lambda):=\int_{0}^{1} e^{-\lambda \frac{y^{(2 \kappa-1) /(1-\kappa)}}{W(y)^{\kappa /(1-\kappa)}}} \frac{d y}{y}
$$

and

$$
\lambda_{J}:=\inf \{\lambda>0 \mid J(\lambda)<\infty\} \in[0, \infty] .
$$

From Theorem 3.1 in [3] applied to $-X$ we see that $\liminf _{t \rightarrow \infty} \frac{X_{t}}{t^{\kappa}}=-\infty$ if and only if $\lambda_{J}=\infty$. Thus $\lambda_{J}=\infty$ implies (3.1).

We now assume, without loss of generality, that $\lambda_{J}<1$.

We will often refer to Proposition 6.2 in the Appendix, where important properties for the function

$$
D(x)=\inf \left\{z>0: \frac{W(z)}{z}=\frac{1}{x^{1-\kappa}}\right\},
$$

for all $x \geq 0$, are obtained.

We proceed to show that (3.1) fails when $\lambda_{J}<1$. First we establish some notation. We will write $X_{t}=X_{t}^{b}+\widetilde{X}_{t}^{b}$, where $X^{b}$ is a spectrally positive Lévy process with jumps bounded by $b$ and $\widetilde{X}^{b}$ is a compensated Poisson process of jumps bigger than $b$. Since $X$ is spectrally positive, a handy bound for $\widetilde{X}_{t}^{b}$ is

$$
\widetilde{X}_{t}^{b}=\sum_{s \leq t} \Delta \widetilde{X}_{t}^{b}-t \int_{b}^{1} x \Pi(d x) \geq-t \int_{b}^{1} x \Pi(d x) .
$$


Also we have

$$
\sup _{t \leq v} R_{t}=\sup _{t \leq v} \sup _{s \leq t}\left(X_{s}-X_{t}\right)=\sup _{t \leq v} \sup _{s \leq t}\left(X_{s}^{D(v)}-X_{t}^{D(v)}+\widetilde{X}_{s}^{D(v)}-\widetilde{X}_{t}^{D(v)}\right),
$$

where by (5.8), (5.4) and Proposition $6.2(b)$ we immediately obtain the bound

$$
\sup _{t \leq v} R_{t} \leq \sup _{t \leq v} \sup _{s \leq t}\left(X_{s}^{D(v)}-X_{t}^{D(v)}\right)+\frac{v W(D(v))}{D(v)}=\sup _{t \leq v} \sup _{s \leq t}\left(X_{s}^{D(v)}-X_{t}^{D(v)}\right)+v^{\kappa} .
$$

Therefore it will suffice to show that

$$
\limsup _{v \rightarrow 0} \frac{\sup _{t \leq v} X_{t}^{D(v)}+\sup _{t \leq v}-X_{t}^{D(v)}}{v^{\kappa}}<\infty \text { a.s. }
$$

For this purpose we use inequality (4.11) in [3], which holds for any zero mean Lévy process with $\sigma=0$. Using this result together with (5.3) gives

$$
\begin{gathered}
P\left(\sup _{t \leq v} X_{t}^{D(v)}>a v^{\kappa}\right) \leq 2 P\left(\left(X_{v}^{D(v)}>a v^{\kappa}-\sqrt{2 v V(D(v))}\right)\right. \\
P\left(\sup _{t \leq v}-X_{t}^{D(v)}>a v^{\kappa}\right) \leq 2 P\left(-X_{v}^{D(v)}>a v^{\kappa}-\sqrt{2 v V(D(v))}\right) .
\end{gathered}
$$

In order to estimate $\sqrt{2 v V(D(v))}$, we use (5.4) together with $(b)$ and $(d)$ from Proposition 6.2 and get $\sqrt{2 v V(D(v))}=o\left(v^{\kappa}\right)$. This means that, for any $\varepsilon>0$ and $v \leq v(\varepsilon)$, we have

$$
\begin{gathered}
P\left(\sup _{t \leq v} X_{t}^{D(v)}>a v^{\kappa}\right) \leq 2 P\left(X_{v}^{D(v)}>(a-\varepsilon) v^{\kappa}\right) \\
P\left(\sup _{t \leq v}-X_{t}^{D(v)}>a v^{\kappa}\right) \leq 2 P\left(-X_{v}^{D(v)}>(a-\varepsilon) v^{\kappa}\right) .
\end{gathered}
$$

For any $a>e+\varepsilon$, we have by Proposition 6.3 that

$$
\max \left\{P\left(\sup _{t \leq v} X_{t}^{D(v)}>a v^{\kappa}\right), P\left(\sup _{t \leq v}-X_{t}^{D(v)}>a v^{\kappa}\right)\right\} \leq e^{-\rho v^{\kappa} / D(v)},
$$

where we have set $\rho=a-\varepsilon-e$. Choose $v_{n}=D^{\leftarrow}\left(1 / 2^{n}\right)$ (see Proposition 6.2 for definition) and use (5.15) above together with Proposition 6.2, part (c), to get

$$
\sum_{n>0} P\left(\sup _{t \leq v_{n}}\left|X_{t}^{D\left(v_{n}\right)}\right|>a v_{n}^{\kappa}\right) \leq 2 K^{-1} \sum_{n>0} K e^{-\rho \frac{\left(1 / 2^{n}\right)(2 \kappa-1) /(1-\kappa)}{W\left(1 / 2^{n}\right)^{\kappa /(1-\kappa)}}},
$$

where $K=\ln 2$. Then setting $q=2^{\frac{2 \kappa-1}{1-\kappa}}$, we see that

$$
\begin{aligned}
& \sum_{n>0} P\left(\sup _{t \leq v_{n}}\left|X_{t}^{D\left(v_{n}\right)}\right|>a v_{n}^{\kappa}\right) \leq K^{-1} \sum_{n>0} K e^{-q \rho \frac{\left(1 / 2^{n+1}\right)^{(2 \kappa-1) /(1-\kappa)}}{W\left(1 / 2^{n}\right)^{\kappa /(1-\kappa)}}} \leq \\
& K^{-1} \int_{0}^{1} e^{-\rho q \frac{y^{(2 \kappa-1) /(1-\kappa)}}{W(y)^{\kappa /(1-\kappa)}}} \frac{d y}{y} .
\end{aligned}
$$


Choose $\rho$, which is the same as choosing $a$, such that $q \rho=1$ and use the fact that $J(1)<\infty$ (recall that $\lambda_{J}<1$ ) to get

$$
\sum_{n>0} P\left(\sup _{t \leq v_{n}}\left|X_{t}^{D\left(v_{n}\right)}\right|>a v_{n}^{\kappa}\right)<\infty
$$

where $a=\frac{1}{q}+\varepsilon+e$. Then the Borel-Cantelli lemma gives (5.10) over $\left\{v_{n}\right\}$, which reads as

$$
\limsup _{n \rightarrow \infty} \frac{\sup _{s \leq v_{n}} R_{s}}{v_{n}^{\kappa}}<\infty \text { a.s. }
$$

Therefore, for any $s \in\left[v_{n+1}, v_{n}\right)$, we have

$$
\begin{aligned}
& \frac{R_{s}}{s^{\kappa}} \leq \frac{\sup _{t \leq v_{n}} R_{t}}{v_{n+1}} \leq \frac{\sup _{t \leq v_{n}} R_{t}}{v_{n}^{\kappa}} \frac{v_{n}^{\kappa}}{v_{n+1}^{\kappa}}=2^{\kappa /(1-\kappa)}\left(\frac{W\left(2^{-n}\right)}{W\left(2^{-n-1}\right)}\right)^{\kappa /(1-\kappa)} \frac{\sup _{t \leq v_{n}} R_{t}}{v_{n}^{\kappa}} \leq \\
& 2^{2 \kappa /(1-\kappa)} \frac{\sup _{t \leq v_{n}} R_{t}}{v_{n}^{\kappa}}
\end{aligned}
$$

where we have made use of the definition of $v_{n}$ and the fact that $\frac{W(x)}{x} \uparrow \infty$, as $x \downarrow 0$. This establishes the theorem.

\section{Appendix}

The first technical result in this Section is the following proposition.

Proposition 6.1. Let $X$ be a Lévy process. Then

$$
\int_{0}^{t} R_{s-} \mathrm{d} \bar{X}_{s}=\sum_{s \leq t} \Delta \bar{X}_{s}(\omega) R_{s-}(\omega) \text { for all } t \text { a.s. }
$$

Proof. Note that $\int_{0}^{t} R_{s-} d \bar{X}_{s}$ is increasing in $t$, so it will be sufficient to show that

$$
\int_{0}^{t} R_{s-} \mathrm{d} \bar{X}_{s}=\sum_{s \leq t} \Delta \bar{X}_{s}(\omega) R_{s-}(\omega) \text { a.s. }
$$

for any fixed $t$. Note that $\bar{X}$ is monotone and fix a path $\omega$. Write the process $\bar{X}$ as

$$
\bar{X}_{u}(\omega)=\sum_{s \leq u} \Delta \bar{X}_{s}(\omega)+G(u, \omega)
$$

where $G(., \omega)$ is nondecreasing and continuous, so that $G(., \omega)$ defines a diffuse measure on $\mathbb{R}_{+}$. Thus

$$
\int_{0}^{t} R_{s-} \mathrm{d} \bar{X}_{s}=\sum_{s \leq t} \Delta \bar{X}_{s}(\omega) R_{s-}(\omega)+\int_{\text {supp } G(\omega) \cap[0, t]} R_{s-} \mathrm{d} G(s, \omega) .
$$


Clearly supp $G(\omega)$ excludes the points in time, $s \in \mathbb{R}_{+}$, at which $\bar{X}_{s}=\bar{X}_{s+h}=\bar{X}_{s-h}$, for some $h>0$. Then we have supp $G(\omega) \subseteq A \cup B \cup C \cup D$ with :

$$
\begin{aligned}
& A=\{s: s \text { is an end or a start point of an excursion }\} \\
& B=\left\{s: \bar{X}_{s-}=\bar{X}_{s-h}<\bar{X}_{s}, \text { for some } h>0\right\} \\
& C=\left\{s: \bar{X}_{s-h}=\bar{X}_{s}<\bar{X}_{s+\delta}, \text { for some } h>0 \text { and any } \delta>0\right\} \\
& D=\left\{s: \bar{X}_{s-}=\bar{X}_{s}>\bar{X}_{s-h}, \text { for any } h>0\right\} .
\end{aligned}
$$

It is immediate that $A$ is countable, since the number of the excursion is countable. $B$ is also countable since by its definition the maximum should be attained by a jump. Finally we see that $C$ is countable by its definition, since it requires a neighbourhood $(s-h, s)$, where no maximum is attained. Using the fact that $G$ is diffuse we get

$$
\int_{A \cap B \cap C \cap[0, t]} R_{s-} \mathrm{d} G(s, \omega)=0 \text { a.s. }
$$

The very definition of $D$ implies, $R_{s-}=0$ on $D$ a.s. This establishes the result.

Proposition 6.2. With $W(x)$ as defined in (5.4) we have $\frac{W(x)}{x}$ is decreasing and $\lim _{x \rightarrow 0} \frac{W(x)}{x}=$ $\infty$. Then $D(x)$ defined in (5.7) has the following properties:

(a) $D(x) \downarrow 0$ as $x \downarrow 0$ and the function is continuous and increasing.

(b) $\frac{W(D(x))}{D(x)}=x^{\kappa-1}$.

(c) $D^{\leftarrow}(x)=\left(\frac{x}{W(x)}\right)^{1 / 1-\kappa}$, where $D^{\leftarrow}(x)$ is the inverse function.

(d) Given that $\lambda_{J}<\infty$ we have $\frac{D(x)}{x^{\kappa}} \rightarrow 0$.

Proof. The result is standard. The proof of (a), (b) and (c) is obvious. For (d) we refer to $[12]$.

Proposition 6.3. For (5.13) and (5.14), and any $a>\varepsilon+e$, we have the following exponential bound:

$$
\max \left\{P\left(X_{v}^{D(v)}>(a-\varepsilon) v^{\kappa}\right), P\left(-X_{v}^{D(v)}>(a-\varepsilon) v^{\kappa}\right)\right\} \leq e^{-\rho v^{\kappa} / D(v)},
$$

where $\rho=a-\varepsilon-e$.

Proof. An application of the Chebyshev inequality to (5.14) yields:

$$
P\left(-X_{v}^{D(v)}>(a-\varepsilon) v^{\kappa}\right)=P\left(e^{-\theta X_{v}^{D(v)}}>e^{\theta(a-\varepsilon) v^{\kappa}}\right) \leq e^{v \int_{0}^{D(v)}\left(e^{-\theta x}+\theta x-1\right) \Pi(d x)-\theta(a-\varepsilon) v^{\kappa}},
$$

for any $\theta>0$. We now use the fact that $e^{-\theta x}+\theta x-1 \leq \theta^{2} x^{2}$ and the definition of $V(x)$ and $W(x)$, to obtain further

$$
P\left(-X_{v}^{D(v)}>(a-\varepsilon) v^{\kappa}\right) \leq e^{v \theta^{2} V(D(v))-\theta(a-\varepsilon) v^{\kappa}} \leq e^{v \theta^{2} W(D(v))-\theta(a-\varepsilon) v^{\kappa}} .
$$

Finally we invoke $(b)$ in Proposition 6.2 , to deduce that

$$
P\left(-X_{t}^{D(v)}>a v^{\kappa}\right) \leq 2 e^{v^{\kappa} \theta^{2} D(v)-\theta(a-\varepsilon) v^{\kappa}},
$$


and setting $\theta=\frac{\gamma}{D(v)}$, with $\gamma>0$, we get

$$
P\left(-X_{t}^{D(v)}>(a-\varepsilon) v^{\kappa}\right) \leq 2 e^{\frac{\gamma v^{\kappa}}{D(v)}(\gamma-(a-\varepsilon))} .
$$

A further application of the Chebyshev inequality to (5.13) with $\theta=\frac{1}{D(v)}$ gives

$$
P\left(e^{\frac{1}{D(v)} X_{v}^{D(v)}}>e^{\frac{1}{D(v)}(a-\varepsilon) v^{\kappa}}\right) \leq e^{v \int_{0}^{D(v)}\left(e^{\frac{x}{D(v)}}-\frac{1}{D(v)} x-1\right) \Pi(d x)-\frac{1}{D(v)}(a-\varepsilon) v^{\kappa}} .
$$

Now, for $u \leq 1$, we have $e^{u}-u-1 \leq e u^{2}$, and therefore

$$
P\left(e^{\frac{1}{D(v)} X_{v}^{D(v)}}>e^{\frac{1}{D(v)}(a-\varepsilon) v^{\kappa}}\right) \leq e^{v e \frac{1}{D(v)^{2}} \int_{0}^{D(v)} x^{2} \Pi(d x)-\frac{1}{D(v)}(a-\varepsilon) v^{\kappa}} .
$$

Recalling the definition of $W(x)$ we see that

$$
\int_{0}^{D(v)} x^{2} \Pi(d x)=V(D(v)) \leq W(D(v))=v^{\kappa-1} D(v)
$$

and hence

$$
P\left(X_{t}^{D(v)}>(a-\varepsilon) v^{\kappa}\right) \leq 2 e^{\frac{v^{\kappa}}{D(v)}(e-(a-\varepsilon))} .
$$

In order to equate the upper bounds in (6.1) and (6.2) set

$$
\gamma(\gamma-(a-\varepsilon))=e-(a-\varepsilon)
$$

and then put $a-\varepsilon=e+\rho$. Thus we get the equation $\gamma^{2}-(e+\rho) \gamma=-\rho$, which clearly has a positive root $\gamma(\rho)$, and choose $\gamma=\gamma(\rho)$ to get the desired result.

Acknowledgement: I am grateful to my supervisor Prof. Ron Doney for his advice and helpful discussions throughout the course of this work, which will also be included in my future $\mathrm{PhD}$ Thesis, see [12]. Also I would like to thank Mr Jacques du Toit for suffering a whole day correcting my English.

\section{References}

[1] Asmussen, S., Avram, F. and Pistorius, M. (2004) Russian and American put options under exponential phase-type Lévy models. Stochastic Process. Appl. 109, No.1, 79-111. MR2024845

[2] Bertoin, J. Lévy Processes. Cambridge University Press, (1996). MR1406564

[3] Bertoin, J., Doney, R. A. and Maller, R. A. (2008) Passage of Lévy processes across power law boundaries at small times. Annals of Probability 36, No.1, 160-197.

[4] Chow, Y. S. and Teicher, H. Probability Theory: Independence, Interchangeability, Martingales. Springer-Verlag 1978. MR0513230

[5] Doney, R. A. and Maller, R. A. (2007) Curve crossing for random walks reflected at their maximum. Ann. Probab. 35, No.1, 1351-1373. MR2330975 
[6] Doney, R. A. and Maller, R. A. (2005) Passage times of random walks and Lévy processes across power law boundaries. Probab. Theory Relat. Fields 133, 57-70. MR2197137

[7] Doney, R. A. (2004) Stochastic bounds for Lévy processes. Ann. Prob. 32, No.2, 1545-1552. MR2060308

[8] Khintchine, A. Ya. (1939) Sur la croissance locale des processes stochastiques homogénes á accroissements indépendants. Izv. Akad. Nauk SSSR 3, 487-508.

[9] Protter P. Stochastic Integration and Differential Equations. A new approach.. SpringerVerlag, first edition, 1990. MR1037262

[10] Rogozin, B. A. (1968) Local behavior of processes with independent increments. Theory Probab. Appl. 16, 575-595 MR0242261

[11] Sato, K. (1999) Lévy Processes and Infinitely Divisible Distributions. Cambridge University Press, Cambridge. MR1739520

[12] Savov, M. Asymptotic behavior of Lévy processes. (2008) Phd Thesis, The University of Manchester, to be submitted

[13] Shepp, L. A. and Shiryaev, A. N. (1993). The Russian Option: reduced regret. Ann. Appl. Probab. 3, No.3, 631-640. MR1233617

[14] Shepp, L. A. and Shiryaev, A. N. (1996). A dual Russian option for selling short. Probability Theory and Mathematical Statistics (A. Ibragimov and A. Yu. Zaitsev, eds.) 209-218. Gordon and Breach, Amsterdam. 